\title{
ORIGINAL ARTICLE \\ Primeros datos sobre migración e hibernación de nóctulos en el Pirineo occidental
}

\author{
Juan Tomás Alcalde ${ }^{1, *}$
}

\author{
${ }^{1}$ Plaza Sabicas, n5, 2ํㅡ - 31015 \\ Pamplona \\ *Corresponding author: \\ jtalcalde@gmail.com \\ DOI: https://doi.org/10.14709/ \\ BarbJ.12.1.2019.07
}

Palabras clave: hibernación, migración, Nyctalus leisleri, Nyctalus noctula, Pirineos.

Keywords: hibernation, migration, Nyctalus leisleri, Nyctalus noctula, Pyrenees.

received: February, 11th 2019 accepted: August, 15th 2019

\begin{abstract}
RESUMEN
La migración de murciélagos en Europa es todavía un aspecto muy poco conocido de su biología. Aunque se conocen desplazamientos migratorios entre el norte y el sur del continente, particularmente en las especies del género Nyctalus, todavía se ignoran sus rutas migratorias. En el período 2016-2018 se estudió la migración post estival de dos especies del género Nyctalus en 22 pasos del Pirineo occidental, utilizando una cámara de vídeo infrarrojo-térmico acoplada a un detector de ultrasonidos. Se grabaron los vuelos de los murciélagos durante las 4-6 primeras horas de la noche, durante 38 noches, y se registraron 175 vuelos rectilíneos de nóctulos, $86 \%$ de los cuales se identificaron como vuelos de entrada a la península ibérica, desde el continente europeo. La mayor parte de ellos (97\%) se asignaron a nóctulos pequeños (Nyctalus leisleri). A pesar de que la cámara utilizada no permitió el estudio cuantitativo de la migración de nóctulos, gracias a este método se pudieron identificar 12 lugares por donde los nóctulos entran a la península. Las mayores tasas de paso se registraron entre el 1 y el 20 de septiembre. Además, se radiomarcaron 10 hembras de nóctulos (5 Nyctalus leisleri y 5 Nyctalus noctula) y mediante radioseguimiento se localizaron dos refugios de hibernación de nóctulos medianos (Nyctalus noctula), ambos en paredes rocosas de cañones del prePirineo. En una de ellas se contabilizaron hasta 31 ejemplares de esta especie. EI conocimiento de las rutas migratorias y los refugios de hibernación son básicos para la adecuada conservación de sus poblaciones.
\end{abstract}




\section{INTRODUCCIÓN}

En las zonas templadas del planeta, muchos animales realizan migraciones anuales para evitar las condiciones más duras del invierno o en busca de alimentación (MilnerGulland et al. 2011). En Europa, una de las principales vías de migración es la que conecta el centro y norte del continente con la península ibérica y África. Aves e insectos atraviesan Europa volando al final del verano, camino de latitudes más bajas. En su trayecto, numerosos ejemplares atraviesan el Pirineo utilizando pasos de montaña (Lack \& Lack 1951, Williams 1957, Bernis 1966). Recientemente se han registrado movimientos migratorios de murciélagos entre el continente europeo y la península ibérica (Ohlendorf et al. 2000, Wohlgemut et al. 2004, Alcalde et al. 2013b) aunque todavía se desconocen algunos detalles como la distribución temporal de estos vuelos, sus rutas o si existen zonas concretas de paso.

El paso de aves por el Pirineo occidental es bien conocido y se prolonga durante el final del verano y el otoño, involucrando a cientos de miles de aves (Bernis 1966). La migración de paseriformes se produce tanto de día como de noche (Zehtindjiev \& Liechti 2003). Las migraciones de murciélagos son, por el contrario, muy poco conocidas debido a la dificultad del estudio de sus vuelos nocturnos.

Hasta la actualidad, la principal fuente de información sobre migraciones de murciélagos procede del anillamiento y recuperación de ejemplares. En Europa se han anillado miles de individuos y con el tiempo se han recogido numerosos datos de desplazamientos migratorios, aunque el número de recuperaciones es muy reducido en comparación con los ejemplares anillados (Hutterer et al. 2005). En la Península ibérica, el anillamiento ha sido prácticamente el único método empleado para conocer movimientos migratorios (Paz et al. 1996, Garin et al. 2008, Alcalde et al. 2013b). No obstante, estos métodos suministran únicamente información de los lugares de captura y recuperación, pero no respecto a las rutas seguidas por los murciélagos, ni su estacionalidad, o repetitividad. El pequeño tamaño de la mayoría de las especies europeas, además, plantea límites a la tecnología actual, al impedir el uso de GPS o emisores de señal satélite (Holland \& Wikelski 2009).

Excepcionalmente, en latitudes elevadas, donde el anochecer es muy paulatino, Ahlén (1997) y Baagøe (2001) mencionan observaciones a simple vista de murciélagos cruzando el mar Báltico en dirección N-S por diferentes puntos de las costas de Suecia y Dinamarca, lo que les permite identificar algunas zonas de paso en otoño. No obstante, en general, todavía se conoce muy poco sobre las dimensiones de la migración de los murciélagos, las fechas en que se produce y las rutas seguidas. El conocimiento de estos datos es muy relevante para asegurar la conservación de estos quirópteros en toda su área de distribución, ya que, por ejemplo, en sus rutas migratorias, algunos murciélagos atraviesan parques eólicos, lo que representa un peligro importante para sus poblaciones (Voigt et al. 2012). Los nóctulos pequeños (Nyctalus leisleri) y medianos (Nyctalus noctula) son migrantes de largas distancias y se encuentran entre las especies más afectadas por los parques eólicos (Rydell et al. 2010). Por ello, y teniendo en cuenta que los collados pirenaicos de Navarra son zonas de paso de numerosas aves migrantes (Bernis 1966) usé una cámara de vídeo infrarrojo-térmico, acoplada a un detector de ultrasonidos para grabar el paso de murciélagos por estas zonas, con el objeto de conocer qué lugares utilizan los murciélagos del género Nyctalus para migrar a través del Pirineo occidental y en qué circunstancias lo hacen. Además, también se valoró la idoneidad de esta metodología para conocer mejor la migración de estos murciélagos, ya que no ha sido utilizada previamente para este fin.

Paralelamente, se radiomarcaron algunas hembras de nóctulos con el objeto de identificar y caracterizar el tipo de refugios que utilizan los individuos migrantes para hibernar, ya que el conocimiento y conservación de estos lugares resulta vital para conservar sus poblaciones. En Europa Central se han identificado algunos refugios de hibernación en árboles, roquedos y construcciones (Dietz \& Kiefer 2017); sin embargo, en la península ibérica apenas se tienen datos de ellos (Alcalde 2010).

\section{MATERIAL Y MÉTODOS}

\section{Filmación de pasos migratorios}

Entre el 6 de agosto y el 28 de octubre (en el trienio 20162018), durante el período migratorio, el paso de nóctulos fue grabado en el extremo occidental del Pirineo. Los puntos de muestreo incluyen 20 collados y dos pasos estrechos del valle del Bidasoa, todos ellos situados, en la región de Navarra (Fig. 1). Algunos collados fueron muestreados en más de una ocasión.

Los collados muestreados se encuentran entre 220 y $1.180 \mathrm{~m}$ de altitud. Los dos pasos estrechos en el valle del Bidasoa se hallan más bajos, entre 20 y 120 m de altitud. Se empleó una cámara de vídeo infrarrojo-térmico (Guide IR517VPro, Wuhuan Guide Infrared Co. Ltd, China) con objetivo angular de $19 \mathrm{~mm}$ (campo de visión: $19.7^{\circ} \mathrm{x}$ $15^{\circ}$ ) acoplado a un detector de ultrasonidos con sistema de división de frecuencias (Pettersson Elektronik D230, Sweden). Mediante esta cámara se puede observar el vuelo de un murciélago hasta una distancia aproximada de 60-70 m en una noche despejada; no obstante, la niebla o un fondo de nubes pueden reducir su efectividad de forma considerable. La cámara ha registrado el vuelo de los murciélagos y también el sonido producido por el detector, colocado al lado. La cámara se tiene que dirigir hacia la zona de la que teóricamente deben provenir los murciélagos (el norte o el este en función de la orientación de las sierras donde se muestree). Durante las tres primeras noches, la cámara se dirigió oblicuamente hacia arriba a nivel experimental (entre 10 y $40^{\circ}$ por encima de la horizontal), pero como únicamente se observaron especies de murciélagos de vuelo bajo (ningún nóctulo), posteriormente se decidió elevarla hasta un ángulo de $75^{\circ} \mathrm{con}$ el fin de registrar el paso de nóctulos migrantes, que a menudo volaban por encima de los $10 \mathrm{~m}$ de altura. De esta forma se grabaron un total de 116,4 horas y se pudo observar la llegada de los murciélagos e identificar los vuelos registrados mediante el análisis de los ultrasonidos grabados simultáneamente. 
Se muestreó durante 38 noches en las que se grabaron las 4-6 primeras horas, dependiendo de la autonomía de la cámara, que fue variable en función de la temperatura ambiental. Se procuró realizar los muestreos en noches con buena climatología (sin lluvia, ni viento intenso, ni temperaturas inferiores a los $10^{\circ} \mathrm{C}$ en el momento de comenzar la grabación) aunque en el Pirineo es frecuente la presencia de fenómenos meteorológicos adversos, por lo que en nueve noches el muestreo tuvo que ser suspendido por la niebla, lluvia o fuerte viento.

Al mismo tiempo se registraron en modo automático los ultrasonidos de los murciélagos que volaban en el entorno de la cámara mediante otro detector de ultrasonidos (Echo Meter Touch 2 Pro, Widlife Acoustics, USA) conectado a una tablet (iPad, Apple, USA) para evaluar la actividad de los murciélagos en cada punto de muestreo y facilitar la identificación in situ de los vuelos grabados en la cámara (Fig. 2).

Los ultrasonidos se analizaron manualmente mediante los siguientes programas (Batsound 4.0, Pettersson Elektronik y Kaleidoscope 4.5.5, Wildlife Acoustics) determinando parámetros típicos de los pulsos, como la frecuencia inicial y final, la frecuencia de máxima intensidad, la duración y el intervalo de tiempo entre ellos, de acuerdo con estudios previos de identificación (Ahlén 1990, Russo \& Jones 2002, Obrist et al. 2004, Barataud 2014).

Los vídeos grabados fueron revisados posteriormente. Se consideraron como desplazamientos migratorios los vuelos realizados en dirección rectilínea de entrada hacia la península ibérica por especies del género Nyctalus. Los murciélagos se identificaron mediante el análisis de los ultrasonidos grabados y teniendo en cuenta también la silueta y el tipo de vuelo de los animales observados (Fig. 3). Los vuelos de nóctulos medianos (Nyctalus noctula) y grandes (Nyctalus lasiopterus) se han agrupado en una sola categoría debido a la dificultad de identificación de algunos de ellos. Los vuelos de murciélagos, realizados en otras direcciones o con trayectorias irregulares no han sido considerados migratorios, aunque se han anotado para conocer la actividad general de la comunidad de murciélagos en estos lugares. También se han descartado los vuelos directos que no han podido ser atribuidos a murciélagos por no haberse escuchado ultrasonidos, ya que podrían ser algunas especies paseriformes que migran durante la noche (Zehtindjiev \& Liechti 2003).

En dos collados de montaña (Izpegi y Artesiaga) se colocaron redes finas en dos noches para determinar algunas que no podían ser identificadas mediante el análisis de ultrasonidos.

\section{Radio-seguimiento de hembras de nóctulo migrantes}

En octubre de 2017 se capturaron 10 hembras de nóctulos en cajas-refugio colocadas en diferentes puntos del centro y norte de Navarra: 5 nóctulos pequeños, Nyctalus leisleri y 5 nóctulos medianos, Nyctalus noctula. Se consideró que estas hembras eran migrantes, ya que, en la mitad norte de la península ibérica, en verano únicamente se han encontrado machos de estas especies (Ibáñez et al.

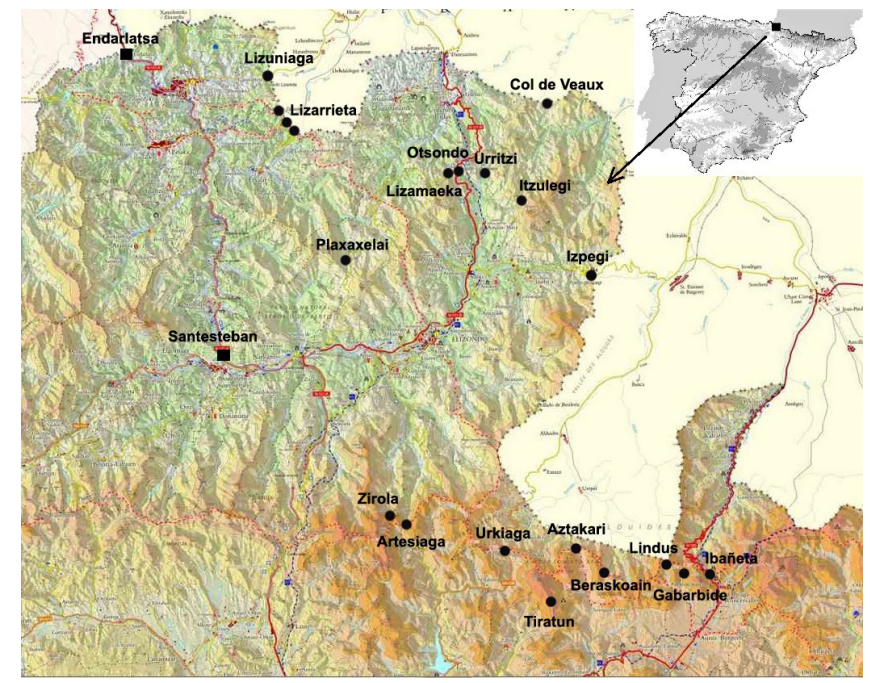

Fig. 1 - Situación de la zona de muestreo, en el Pirineo occidental. Collados de montaña (círculos) y pasos estrechos de valles (cuadrados) donde se ha llevado a cabo el estudio. Fuente: SITNA, Gobierno de Navarra.// Location of the study area, in Western Pyrenees. Mountain passes (circles) and narrow valley passes (squares) where the study has been carried out. Source: SITNA, Navarra's Government.

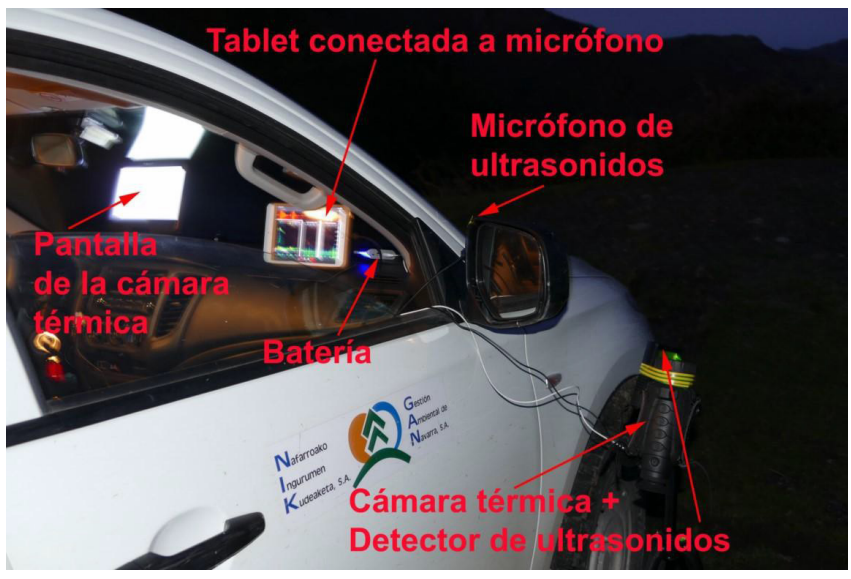

Fig. 2 - Material empleado para la grabación de los vuelos y de los ultrasonidos de los murciélagos. // Material used for recording flights and ultrasounds of bats.

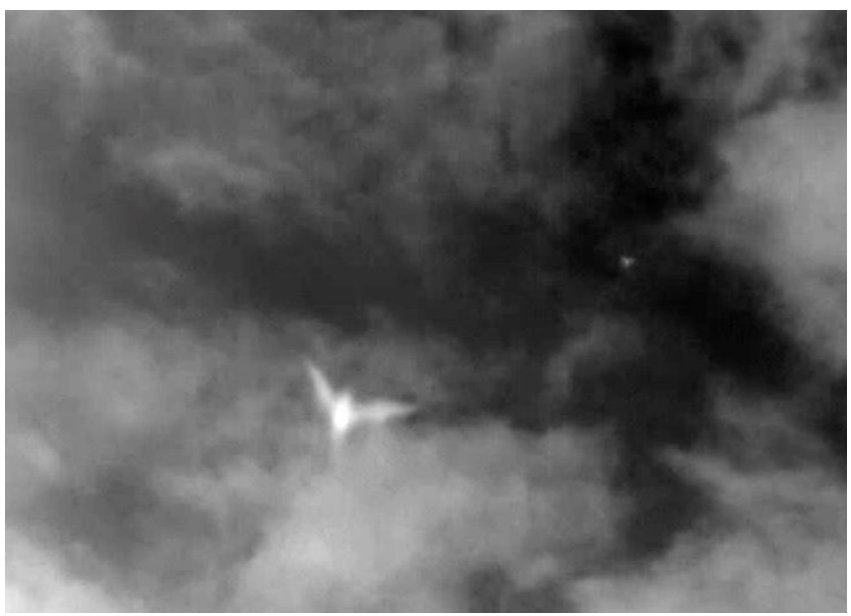

Fig. 3 -Nóctulo mediano N. noctula registrado en la cámara de infrarrojo-térmico y escuchado en el detector de ultrasonidos. Más arriba a la derecha pasa otro animal silencioso y volador sin identificar. Los animales sin identificar han sido descartados del análisis. // Common noctule, N. noctula recorded in the infrared-thermal image camera and heard in the ultrasound detector. Further up on the right, a silent and unidentified flying animal passes by. Unidentified animals have been discarded from the analysis. 
2009; datos propios para Navarra). Estos ejemplares fueron marcados con radioemisores (Pip3, Biotrack Ltd., UK) y se liberaron posteriormente para tratar de encontrar sus refugios de hibernación. Se marcaron varios individuos, ya que existía la posibilidad de que se perdiera la pista de algunos de ellos debido a la dificultad de su localización, particularmente al tratarse de especies que realizan grandes desplazamientos. Empezamos a buscar los nóctulos la misma semana de su marcaje y se realizaron prospecciones posteriores semanalmente con un radio receptor SIKA y una antena yagi de tres elementos (Biotrack Ltd., UK). La búsqueda fue intensiva en un radio de $30 \mathrm{~km}$ alrededor de los lugares de captura y en áreas concretas más alejadas donde se ya se conocía la presencia de nóctulos, hasta un máximo de $200 \mathrm{~km}$. Los refugios identificados fueron caracterizados y visitados periódicamente cada 15 días para comprobar si los murciélagos marcados seguían allí, hasta el 30 de marzo, cuando se estimó que las baterías se habían agotado.

\section{RESULTADOS}

En total registramos 175 vuelos rectilíneos de nóctulos: 151 (86\%) vuelos de entrada en la Península ibérica, en dirección oeste o sur y 24 (14\%) de salida, hacia el este o el norte. El $97 \%$ de los vuelos de entrada fueron de nóctulos pequeños ( $N$. leisleri). El resto correspondió al grupo de nóctulos medianos ( $N$. noctula) o grandes ( $N$. lasiopterus). Considerando el total de horas grabadas con la cámara dirigida hacia el cielo, se registraron 1,2 vuelos de nóctulos por hora.

En los collados de montaña, el paso migratorio de murciélagos fue muy irregular: sólo se registró paso de nóctulos en 20 noches (53\%). El 97\% de los vuelos directos de nóctulos fue de entrada hacia la península, en dirección sur u oeste; tan sólo el 3\% fue en dirección de salida, hacia el norte o el este. Se observaron vuelos de entrada entre el 23 de agosto y el 19 de octubre, con una mayor intensidad entre el 1 y el 20 de septiembre (Fig. 4). Considerando las grabaciones realizadas en las cinco primeras horas de la noche en estos collados, la tasa de vuelos/hora fue significativamente más intensa en la tercera y cuarta hora tras el ocaso $\left(\chi_{4}^{2}=26,182, p<0,001\right)$.

En los pasos estrechos de fondo de valle, los vuelos directos de entrada de nóctulos constituyeron el 63\% de los registrados; el $37 \%$ restante se identificaron como vuelos de salida.

En total, se registró la entrada de ejemplares migrantes por 10 collados de montaña y dos pasos estrechos de valles. En la tabla 1 se indican las fechas y lugares con mayor tasa de vuelos/hora. Todos los vuelos grabados fueron de individuos aislados, salvo en tres ocasiones, donde se observaron parejas de nóctulos pequeños volando uno tras otro.

En todo el muestreo se grabaron 12.026 archivos de ultrasonidos de murciélagos (81,9 vuelos/hora) y se identificaron 15 especies, además de ejemplares de los géneros Myotis y Plecotus que no pudieron ser identificados hasta nivel de especie. Todos los puntos de muestreo salvo uno (Tiratun) mostraron actividad de murciélagos. Las especies más frecuentes (con mayor número de grabaciones de ultrasonidos) fueron el murciélago enano (Pipistrellus pipistrellus) con el $52 \%$ de los vuelos y el nóctulo pequeño (Nyctalus leisleri) con el 31\%. Cabe destacar la elevada actividad y diversidad encontrada en el collado de Artesiaga, donde se identificaron 17 especies mediante análisis de ultrasonidos y capturas con red y donde se llegaron a contabilizar 192 vuelos/h en septiembre de 2016.

De las 10 hembras de nóctulos marcadas con radioemisores, sólo dos fueron localizadas durante el invierno. Las dos eran ejemplares de nóctulos medianos ( $N$. noctula) capturados en Javier $\left(42^{\circ} 35^{\prime} \mathrm{N}, 1^{\circ} 13^{\prime} \mathrm{O}\right)$. Una de ellas se localizó posteriormente dentro de la Foz de Lumbier

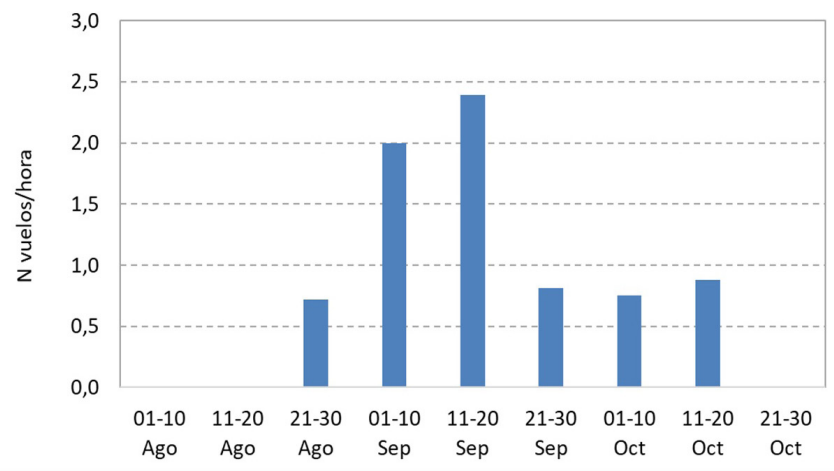

Fig. 4 - Tasas de vuelos de entrada de nóctulos por los collados de montaña por hora de grabación, cada diez días. // Rates of entry flights of noctules by the mountain passes per hour, each ten days.

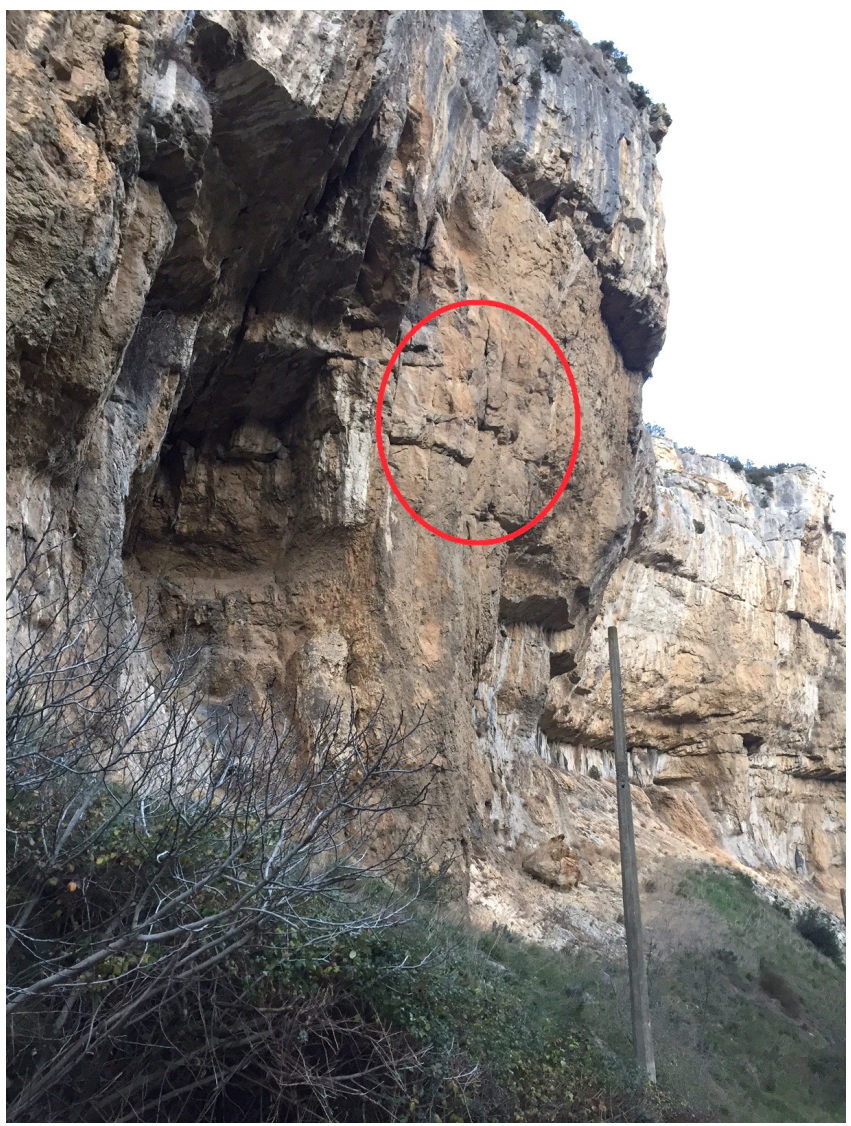

Fig. 5 - Grietas donde hibernó un nóctulo mediano radiomarcado. En grietas de esta zona se observó la salida de 31 nóctulos medianos el 30/03/2018.// Cracks where a radio-tagged common noctule hibernated. In cracks of this area, 31 common noctules were observed flying out on 30/03/2018. 
Tabla 1. Fechas y lugares donde se registraron las mayores tasas de vuelos de entrada por hora de grabación. * Izpegi fue muestreado en dos ocasiones.// Dates and places where the highest entry-flights rates have been observed per recorded hour. * Izpegi was sampled twice.

\begin{tabular}{llcccccc}
\hline Fecha & Lugar & $\begin{array}{c}\text { Tipo de } \\
\text { paso }\end{array}$ & $\begin{array}{c}\text { Vuelos } \\
\text { entrada }\end{array}$ & $\begin{array}{c}\text { Vuelos } \\
\text { salida }\end{array}$ & $\begin{array}{c}\text { Balance de vuelos } \\
\text { de entrada }\end{array}$ & $\begin{array}{c}\text { Horas de } \\
\text { grabación }\end{array}$ & Tasa v/h \\
\hline $24 / 08 / 2017$ & Izpegi ${ }^{*}$ & Collado & 14 & 0 & 14 & 3,5 & 4,0 \\
$07 / 09 / 2017$ & Artesiaga & Collado & 12 & 0 & 12 & 3,8 & 3,2 \\
$12 / 09 / 2017$ & Izpegi & Collado & 36 & 0 & 36 & 3,5 & 10,3 \\
$20 / 09 / 2017$ & Ibañeta & Collado & 9 & 0 & 9 & 4,3 & 2,1 \\
$23 / 09 / 2017$ & Lizuniaga & Collado & 9 & 0 & 9 & 5,8 & 1,6 \\
$02 / 09 / 2018$ & Endarlatsa & Valle & 21 & 7 & 14 & 5,0 & 2,8 \\
Total & & & $\mathbf{1 0 1}$ & $\mathbf{7}$ & $\mathbf{9 4}$ & $\mathbf{2 5 , 9}$ & $\mathbf{3 , 6}$ \\
\hline
\end{tabular}

$\left(42^{\circ} 38^{\prime} \mathrm{N}, 1^{\circ} 18^{\prime} \mathrm{O}\right)$, a $7,5 \mathrm{~km}$ del lugar de marcaje. Esta hembra permaneció en una grieta de una pared rocosa con orientación oeste a una altura de $8 \mathrm{~m}$, al menos desde el $1 / 12 / 2017$ hasta el 14/3/2018 (Fig. 5). A finales de marzo, con la batería teóricamente gastada, ya no se escuchó. El 30 de marzo de 2018 se observó la salida de 31 nóctulos medianos desde diferentes grietas de este roquedo, cercanas a la zona donde había hibernado la hembra marcada. La otra hembra se localizó en un roquedo inaccesible de la Foz de Arbaiun $\left(42^{\circ} 40^{\prime} \mathrm{N}, 1^{\circ} 11^{\prime} \mathrm{O}\right)$, con orientación sur, a 9,6 km de su lugar de marcaje. Mediante revisiones quincenales, se comprobó que permaneció allí al menos desde el 23/10/2017 hasta el 10/2/2018. Ambas hembras hibernaron más al norte del lugar donde habían sido capturadas.

Del resto de individuos marcados, dos hembras de nóctulos pequeños fueron localizadas durante el primer mes siguiente a su marcaje en cajas-refugio de la misma zona donde habían sido capturadas, aunque después se perdió su pista. Un emisor de estas fue localizado posteriormente en el suelo. El resto de hembras no fue nunca localizado tras su marcaje.

\section{DISCUSIÓN}

Los murciélagos migrantes son uno de los grupos peor conocidos de entre los taxones que realizan movimientos migratorios (Holland \& Wikelski 2009). Sus vuelos nocturnos y sus poblaciones relativamente pequeñas en comparación con otros grupos de animales migrantes, como aves o insectos, dificultan en gran medida su estudio. En Europa, el anillamiento y recuperación de ejemplares ha sido, hasta fechas recientes, el método más utilizado y eficaz para avanzar en el conocimiento de la migración de estos mamíferos (Hutterer et al. 2005). También las observaciones de la presencia estacional de especies migrantes en zonas concretas o la variación de la sex-ratio de sus poblaciones han permitido inferir patrones de desplazamiento (Sluiter \& Van Heerdt 1966, Strelkov 2000, Ibáñez et al. 2009). Recientemente se han sumado nuevas técnicas como la radiotelemetría, el radar, el uso de marcadores genéticos y el estudio de isótopos estables (Petit \& Mayer 2000, Holland \& Wikelski 2009, Moussy et al. 2013, Popa-Lisseanu et al. 2012, Voigt et al. 2012) que han provisto de datos sobre el origen de murciélagos migrantes capturados en otoño.
En este trabajo se ha observado una proporción muy desigual entre las especies de nóctulos identificados en los pasos de montaña, ya que el $97 \%$ son nóctulos pequeños y las otras dos especies sólo proporcionan, conjuntamente, el $3 \%$ de los registros restantes. Ello parece indicar que los nóctulos pequeños constituyen el principal contingente de murciélagos migrantes en el Pirineo occidental, hecho reforzado además por su distribución en la mitad septentrional de la península ibérica, donde hay zonas extensas en las que es una especie relativamente frecuente (Agirre-Mendi 2007, Alcalde et al. 2008), en comparación con los nóctulos medianos y grandes, mucho más escasos en el mismo territorio (Alcalde 2007, Juste 2007, Alcalde et al. 2008). En concordancia con las escasas observaciones de nóctulos grandes realizadas en el presente trabajo, Ibáñez et al. (2009) ponen en duda el carácter migrante de esta especie, ya que las colonias reproductoras conocidas en Europa occidental se encuentran únicamente en el sur del continente.

La situación del nóctulo mediano es aún incierta: el grueso reproductor de su población se encuentra en el centro y norte de Europa (Bogdanowicz 1999) y en la península ibérica sólo se conoce una colonia reproductora, situada en Aranjuez (Ruedi et al. 1998). Además, en el norte de la península se han localizado varios grupos formados únicamente por machos en verano (Alcalde 2007, Flechoso et al. 2018), a los que acuden hembras tras la reproducción, pero todavía se desconoce el lugar de procedencia de estas. Estas observaciones, junto con los desplazamientos de ejemplares entre el norte y centro del continente y el centro y sur de Francia (Hutterer et al. 2005) permiten sospechar que en la península ibérica puedan confluir poblaciones sedentarias de esta especie junto con otras migrantes. También hay que considerar que la escasez de vuelos registrados de nóctulos medianos y grandes podría deberse al insuficiente alcance de la cámara térmica utilizada, ya que ambas especies realizan vuelos a gran altura con frecuencia (Dietz \& Kiefer 2017).

Los únicos datos concretos de movimientos migratorios de nóctulos entre la península ibérica y Centroeuropa proceden de nóctulos pequeños anillados y recuperados posteriormente: dos hembras anilladas en verano en Alemania fueron recuperadas posteriormente en Burgos 
y Madrid (Ohlendorf et al. 2000, Wohlgemut et al. 2004); además, una hembra anillada en otoño en el norte de Navarra y que hibernó ese invierno en una caja-refugio de la misma zona, fue hallada posteriormente en Bélgica (Alcalde et al. 2013b). Por otro lado, la revisión en otoño de cajas-refugio colocadas en algunos bosques de Navarra ha revelado una elevada ocupación de las mismas por machos y hembras de nóctulos pequeños, y en menor proporción, también por ejemplares de ambos sexos de nóctulos medianos y grandes, lo que sugiere la llegada de ejemplares (al menos hembras, pero es probable que también algunos machos) de estas especies tras la reproducción (Alcalde et al. 2013a).

La grabación de la llegada de más de un centenar de nóctulos pequeños por 10 collados de montaña y dos pasos estrechos de valles del norte de Navarra, provenientes de latitudes superiores, ha permitido identificar zonas de paso por el Pirineo y conocer algunos detalles de estas llegadas. Estas se dan preferentemente de forma individual, sin formar bandos. Ahlén (1997) detectó vuelos individuales de varias especies de murciélagos en dirección sur o suroeste en la costa meridional Suecia, aunque al igual que Baagøe (2001) también mencionó la observación de grupos de nóctulos medianos migrando en bandos, al parecer tras la acumulación de ejemplares en la costa debido a períodos de viento intenso. Por el momento, en el Pirineo no se han observado estos bandos, aunque no son descartables, ya que en dos ocasiones se ha detectado la desaparición de grupos de nóctulos pequeños y medianos que estaban asentados en cajas-refugio cercanas y las abandonaron repentinamente en una noche (Alcalde, obs. pers).

Algunas zonas parecen tener un mayor paso de murciélagos, destacando el collado de Izpegi, donde una noche se observó una tasa de vuelos/hora mucho más elevada que en el resto de las zonas (Tabla 1). No obstante, no es posible realizar comparaciones precisas entre el paso observado por diferentes collados, ya que han sido muestreados en fechas diferentes y con distintas condiciones climáticas. La llegada parece prolongada en el tiempo, distribuyéndose al menos desde el 20 de agosto hasta el 20 de octubre. Los valores más elevados han sido registrados entre el 1 y 20 de septiembre, lo cual coincide aproximadamente con observaciones recogidas por otros autores: en La Rioja, las hembras de nóctulos pequeños comienzan a aparecer a partir de mediados de agosto y se alcanza el $50 \%$ del sex ratio en septiembre (Ibáñez et al. 2009); además, la hembra reproductora anillada en verano en Alemania, fue recuperada en Burgos el 28/09/1999 (Ohlendorf et al. 2000).

La sospecha de que los vuelos directos de nóctulos en dirección de entrada a la península son en su gran mayoría movimientos migratorios, se ve apoyada por la notable diferencia observada en cuanto a la direccionalidad de estos vuelos sobre los collados, ya que únicamente $3 \%$ de ellos fueron de retorno hacia el continente europeo. Se podría sospechar que los nóctulos con vuelo directo cruzando los collados estaban únicamente acudiendo a cazar a otro valle; sin embargo, el registro de una mayor tasa de pasos en la tercera y cuarta hora tras el ocaso, permiten descartar esa posibilidad, ya que en esa circunstancia, se habría observado un mayor número de vuelos en la primera hora tras el ocaso. De forma similar, Ahlén (1997) observa vuelos de diferentes especies de murciélagos que al atardecer abandonan la costa meridional de Suecia en dirección sur y suroeste, lo que interpreta como desplazamientos migratorios. Por el contrario, los vuelos observados en valles son más difíciles de interpretar, porque esa diferencia es menor. La observación de un $37 \%$ de vuelos "de salida", es decir, en dirección norte, indica que el lugar de muestreo (Endarlatsa) es probablemente una zona de campeo de esta especie, en la que se producen muchas idas y venidas constantemente.

Aunque la cámara utilizada ha permitido concretar diversas zonas de paso de nóctulos migrantes, no resulta un método eficaz para realizar estimaciones cuantitativas de la migración por esos lugares, como demuestra el reducido número de observaciones en relación al esfuerzo realizado y a los vuelos registrados mediante grabadora de ultrasonidos. Ello parece deberse a que el micrófono de ultrasonidos puede registrar los vuelos producidos en todo su entorno cercano, mientras que la cámara de vídeo graba un campo de visión considerablemente más reducido, a pesar de haberse utilizado un objetivo angular. Además, la cámara resulta ineficaz cuando hay niebla, fenómeno frecuente en los puntos muestreados, mientras que el micrófono de ultrasonidos sigue registrando vuelos en esas condiciones.

Por otro lado, cabe destacar la elevada actividad de murciélagos en estos collados y valles, así como la gran diversidad de especies identificadas mediante las grabaciones de ultrasonidos, particularmente en el collado de Artesiaga. Ello hace suponer que algunos de estos collados son zonas de caza de numerosas especies de murciélagos, que pueden aprovechar el paso o el arrastre de insectos por estas zonas estrechas. Artesiaga además es una zona de pastos que acoge ganado ovino, vacuno y caballar, lo cual atrae particularmente la actividad de los murciélagos (Downs \& Sanderson 2010).

No es de extrañar la localización de dos hembras de nóctulos medianos hibernando en roquedos, ya que también anteriormente se habían encontrado ejemplares de esta especie hibernando en Etxauri, otro roquedo situado en el oeste de Navarra (Alcalde 2010) y también en roquedos del continente europeo (Dietz \& Kiefer 2017). Al menos una de las zonas identificadas en este trabajo (Foz de Lumbier) es refugio de un grupo de hibernación de esta especie, aunque no es posible determinar si se trata de murciélagos migrantes o sedentarios, más aún si se tiene en cuenta que las dos hembras localizadas hibernaron en puntos situados más al norte de las zonas donde fueron marcadas.

La pérdida de recepción de ocho nóctulos radiomarcados, a pesar de un intenso esfuerzo prospectivo, podría deberse a que estos ejemplares han continuado su migración más al sur, fenómeno confirmado por las observaciones de Ohlendorf et al. (2000) y Wohlgemut et al. (2004), con el hallazgo de ejemplares migrantes en Burgos y Madrid de nóctulo pequeño.

Dado que numerosos nóctulos pequeños y medianos realizan movimientos migratorios de larga distancia (Hutterer et al. 2005), su conservación requiere considerar 
grandes áreas geográficas. El conocimiento de las rutas migratorias de estos murciélagos y la identificación de refugios de hibernación permitirá desarrollar medidas adecuadas para la conservación de estas especies y de sus hábitats.

\section{AGRADECIMIENTOS}

Agradecemos la ayuda prestada por Carlos Astrain, Ainhoa Isturiz, Joseba Oroz y Mikel Garate de Nafarroako Ingurumen Kudeaketa SA - Gestión Ambiental de Navarra SA, del Gobierno de Navarra en el trabajo de campo y la revisión del manuscrito original. Así mismo agradecemos a Karmele Areta y Gloria Giralda del Servicio de Medio Natural del Gobierno de Navarra por impulsar el estudio y la conservación de los murciélagos en la zona pirenaica. Además, un eficiente grupo de guardas forestales del Departamento de Desarrollo Rural, Medio Ambiente y Administración Local del Gobierno de Navarra ha ayudado a prospectar y localizar los nóctulos radiomarcados.

Se agradecen también las aportaciones realizadas por dos revisores anónimos para mejorar el manuscrito original.

Este trabajo se ha realizado dentro del Proyecto Lindus / 2 perteneciente al Programa Interreg POCTEFA, sufragado por el Fondo Europeo de Desarrollo Regional (FEDER).

\section{BIBLIOGRAFÍA}

AGIRRE-MENDI, P. T. (2007). Nyctalus leisleri (Kuhl, 1817). In: Atlas y Libro Rojo de los Mamíferos Terrestres de España. ed.: Dirección General para la Biodiversidad - SECEM SECEMU. Madrid, España, p.222-225.

AHLÉN, I. (1990). Identification of bats in flight. ed.: Swedish Society for Conservation of Nature \& Swedish Youth Association for Environmental Studies and Conservation. Stockholm, Sweden, 50 pp.

AHLÉN, I. (1997). Migratory behaviour of bats at south Swedish coasts. Z. Säugetierkunde, 62: 375-380.

ALCALDE, J. T. (2007). Nyctalus noctula (Schreber, 1774). In: Atlas y Libro Rojo de los Mamíferos Terrestres de España. ed.: Dirección General para la Biodiversidad - SECEM SECEMU. Madrid, España, p.228-230.

ALCALDE, J. T., TRUJILLO, D., ARTÁZCOZ, A. \& AGIRRE-MENDI, P. T. (2008). Distribución y estado de conservación de los quirópteros en Aragón. Graellsia, 64(1): 3-16. https:// doi.org/10.3989/graellsia.2008.v64.i1.51

ALCALDE, J. T. (2010). Fenología de Nyctalus noctula en Pamplona. III Jornadas de la Asociación para la Conservación y el Estudio de los Murciélagos (SECEMU). 21-22 de noviembre. La Coruña, España.

ALCALDE, J. T., CAMPION, D., FABO, J., MARÍN, F., ARTÁZCOZ, A., MARTÍNEZ, I. \& ANTÓN, I. (2013a). Ocupación de cajas-refugio por murciélagos en Navarra. Journal of bat research \& conservation, 6(1): 35-45. https://doi. org/10.14709/BarbJ.6.1.2013.05
ALCALDE, J. T., IBÁÑEZ, C., ANTÓN, I. \& NYSSEN, P. (2013b). First case of migration of a Leisler's bat (Nyctalus leisleri) between Spain and Belgium. Le Rhinolophe, 19: 87-88.

BAAG $\varnothing E$, H. J. (2001). Danish bats (Mammalia: Chiroptera): Atlas and analysis of distribution, occurrence and abundance. Steenstrupia 26(1): 1-117.

BARATAUD, M. (2014). Écologie acoustique des chiroptères d'Europe. Identification des espèces, étude de leurs habitats et comportements de chasse. ed.: Biotope Mèze - Muséum National d'Histoire Naturelle. Paris, France, 344 pp.

BERNIS, F. (1966). Migración en aves. Tratado teórico y práctico. ed.: Sociedad Española de Ornitología. Madrid, España, 486 pp.

BOGDANOWICZ, W. (1999). Nyctalus noctula (Schreber, 1774). In: The atlas of european mammals. ed.: Societas Europaea Mammalogica. London, United Kingdom, p.136-137.

DIETZ, C. \& KIEFER, A. (2017). Los murciélagos de Europa. ed.: Omega. Barcelona, España, 400 pp.

DOWNS, N. C. \& SANDERSON, L. J. (2010). Do bats forage over cattle dung or over cattle? Acta Chiropterologica, 12(2): 349-358. https://doi.org/10.3161/150811010X537936

FLECHOSO, M. F., FERNÁNDEZ, D. \& ALCALDE, J. T. (2018). Primera colonia de nóctulos medianos (Nyctalus noctula Schreber, 1774) en Castilla y León (Norte de España). Journal of bat research \& conservation, 11(1). https://doi.org/10.14709/barbj.11.1.2018.02

GARIN, I., AIHARTZA, J., AGIRRE-MENDI, P. T., ALCALDE, J. T., DE LUCAS, J., DE PAZ, O., GOITI, U. \& ARTÁZCOZ, A. (2008). Seasonal movements of the Schreibers' bat, Miniopterus schreibersii, in the northern Iberian Peninsula. Italian Journal of Zoology, 75(3): 263-270. https://doi.org/10.1080/11250000801927850

HOLLAND, R. A. \& WIKELSKI, M. (2009). Studying the migratory behavior of individual bats: current techniques and future directions. Journal of Mammalogy, 90(6): 1324-1329. https://doi. org/10.1644/09-MAMM-S-086R2.1

HUTTERER, R., IVANOVA, T., MEYER-CORDS, C. \& RODRIGUES, L. (2005). Bat migrations in Europe. A review of banding and data literature. ed.: Federal Agency for Nature Conservation. Bonn, Germany, 162 pp.

IBÁÑEZ, C., GUILLÉN, A., AGIRRE-MENDI, P. T., JUSTE, J., SCHREUR, G., CORDERO, A. I. \& POPA-LISSEANU, A. G. (2009). Sexual segregation in Iberian Noctule bats. Journal of Mammalogy, 90(1): 235-243. https://doi. org/10.1644/08-MAMM-A-037.1

JUSTE, J. (2007). Nyctalus lasiopterus (Schreber, 1780). In: Atlas y Libro Rojo de los Mamíferos Terrestres de España. ed.: Dirección General para la Biodiversidad - SECEM SECEMU. Madrid, España, p.233-235. 
LACK, D. \& LACK, E. (1951). Migration of insects and birds through a Pyrenean pass. Journal of Animal Ecology, 20(1): 63-67. https://doi.org/10.2307/1644

MILNER-GULLAND, E. J., FRYXELL, J. M. \& SINCLAIR, A. R. E. (2011). Animal migration. A synthesis. ed.: Oxford University Press. New York, EEUU, 268 pp. https://doi. org/10.1093/acprof:oso/9780199568994.001.0001

MOUSSY, C., HOSKEN, D. J., MATHEWS, F., SMITH, G. C., AEGERTER, J. N. \& BEARHOP, S. (2013). Migration and dispersal patterns of bats and their influence on genetic structure. Mammal Review, 43(3): 183-195. https://doi. org/10.1111/j.1365-2907.2012.00218.x

OHLENDORF, V. B., HECHT, B., STRASSBURG, D. \& AGIRREMENDI, P. T. (2000). Fernfund eines Kleinabendseglers (Nyctalus leisleri) in Spanien. Nyctalus (N.F.), 7(3): 239242.

OBRIST, M. K., BOESCH, R. \& FLÜCKIGER, P. F. (2004). Variability in echolocation call design of 26 Swiss bat species: consequences, limits and options for automated field identification with a synergetic pattern recognition approach. Mammalia, 68(4): 307-322. https://doi.org/10.1515/mamm.2004.030

PAZ, O. DE, FERNÁNDEZ, R. \& BENZAL, J. (1996). El anillamiento de quirópteros en el centro de la Península Ibérica durante el periodo 1977-1986. Boletín de la Estación Central de Ecología, 30: 113-138.

PETIT, E. \& MAYER, F. (2000). A population genetic analysis of migration: the case of the noctule bat (Nyctalus noctula). Molecular Ecology, 9(6): 683-690. https://doi. org/10.1046/j.1365-294x.2000.00896.x

POPA-LISSEANU, A. G., SÖRGEL, K., LUCKNER, A., WASSENAAR, L. I., IBÁÑEZ, C., KRAMER-SCHADT, S., CIECHANOWSKI, M., GÖRFÖL, T., NIERMANN, I., BEUNEUX, G. et al. (2012). A triple-isotope approach to predict the breeding origins of European bats. PLOS One, 7(1): e30388. https://doi.org/10.1371/journal. pone. 0030388
RUEDI, M., TUPINIER, Y. \& PAZ, O. DE. (1998). First breeding record for the noctule bat (Nyctalus noctula) in the Iberian Peninsula. Mammalia, 62: 301-304.

RUSSO, D. \& JONES, G. (2002). Identification of twentytwo bat species (Mammalia: Chiroptera) from Italy by analysis of time-expanded recordings of echolocation calls. Journal of Zoology (London), 258(1): 91-103. https://doi.org/10.1017/S0952836902001231

RYDELL, J., BACH, L., DUBOURG-SAVAGE, M.-J., GREEN, M., RODRIGUES, L. \& HEDENSTRÖM, A. (2010). Bat mortality at wind turbines in northwestern Europe. Acta Chiropterologica, 12(2): 261-274. https://doi. org/10.3161/150811010X537846

SLUITER, J. W. \& VAN HEERDT, P. F. (1966). Seasonal habits of the noctule bat (Nyctalus noctula). Archives Néerlandaises de Zoologie, 16(4): 423-439. https://doi. org/10.1163/036551666X00011

STRELKOV, P. P. (2000). Seasonal distribution of migratory bat species (Chiroptera, Vespertilionidae) in eastern Europe and adjacent territories: nursing area. Myotis, 37: 7-25.

VOIGT, C. C., POPA-LISSEANU, A. G., NIERMANN, I. \& KRAMER-SCHADT, S. (2012). The catchment area of wind farms for European bats: A plea for international regulations. Biological Conservation, 153: 80-86. https:// doi.org/10.1016/j.biocon.2012.04.027

WILLIAMS, C. B. (1957). Insect migration. Annual Review of Entomology, 2: 163-180. https://doi.org/10.1146/ annurev.en.02.010157.001115

WOHLGEMUT, R., DEVRIENT, I., GARCÍA, A. \& HUTTERER, R. (2004). Long-distance flight of a Lesser noctule (Nyctalus leisleri) after rehabilitation. Myotis, 41-42: 69-73.

ZEHTINDJIEV, P. \& LIECHTI, F. (2003). A quantitative estimate of the spatial and temporal distribution of nocturnal bird migration in south-eastern Europe - a coordinated moon-watching study. Avian Science, 3(1): 37-45. 